\title{
EIN GENETISCHER ALGORITHMUS FÜR DAS TOURENPLANUNGSPROBLEM
}

\author{
Herbert Kopfer, Universität Bremen \\ Elmar Erkens, Universität Bremen \\ Giselher Pankratz, Wilnsdorf
}

Die Aufgaben bei der Entwicklung eines Genetischen Algorithmus (GA) bestehen in der Definition einer geeigneten Problemrepräsentation, der Konfigurierung des GA, der Implementierung und anschlieBender Parametrisierung.

Die hier gewählte Repräsentation von Tourenplänen durch Strings beruht auf Permutationen der Kundenorte und ist auf praktische Problemstellungen erweiterbar. In einer ersten Version des GA ordnet ein einfacher nachgeschalteter Clusterbuilder die Orte einer Permutation nach dem FIFO-Prinzip den verfügbaren Fahrzeugen zu. Die Reihung der Orte (Route) einer Tour entspricht dabei der Reihenfolge, in der die Orte in der Permutation auftreten. Außerdem wurde eine zweite Version des Clusterbuilders entwickelt, bei der zur Dekodierung der Permutation das Prinzip des Savings-Verfahrens ausgenutzt wird (intelligenter Clusterbuilder).

Bei der Konfigurierung von GAs sind Entwurfsentscheidungen bezüglich des Reproduktionsmodells, der Selektionstechnik und der Rekombinationsoperatoren zu treffen. Als Reproduktionsmodell wurde das Steady-State-Modell, zur Selektion ein ranking-basiertes Verfahren und zur Rekombination problemangejaßte permutationsbewahrende Crossov'sr- und Mutationsoperatoren gewählt. Das Verfahrea wurde in Turbo Pascal 6.0 implementiert. Zur Parametrisierung wurden geeignete Werte für die Populationsgröße, Crossover- und Muationswahrscheinlichkeit sowie weitere Parameter zur Steuerung des Selektionsmechanismus empirisch ermittelt. Eine Interpolation der Parameter wurde in die Untersuchung einbezogen.

Bei beiden obigen Repräsentationen durchläuft der GA einen Lemprozeß und setzt sich deutlich von der reinen Zufallssuche ab. Bei Verwendung des ersten (primitiven) Clusterbuilders läßt sich die Lösungsqualität verbessern, indem in Anlehnung an das Sweep-Verfahren einige gute Permutationen erzeugt und in die Anfangspopulation eingepflanzt werden (Hybridisierung durch Seeding). Der auf dem Savings-Prinzip basierende Clusterbuilder ist den beiden anderen Varianten der Repräsentation deutlich überlegen und zeigt beim Test an Hand von Literaturbeispielen ein sehr gutes Lösungsverhalten.

Im Hinblick auf die Praxisrelevanz wurde die Zielsetzung der reinen Streckenminimierung durch die Minimierung der Kosten aufgrund einer gestuften Kostenfunktion ersetzt. Die dazu erforderliche Anpassung des GA war durch eine Modifikation der Evaluierungsfunktion leicht zu realisieren. Das Lernverhalten des GA blieb davon unbeeinflußt. 